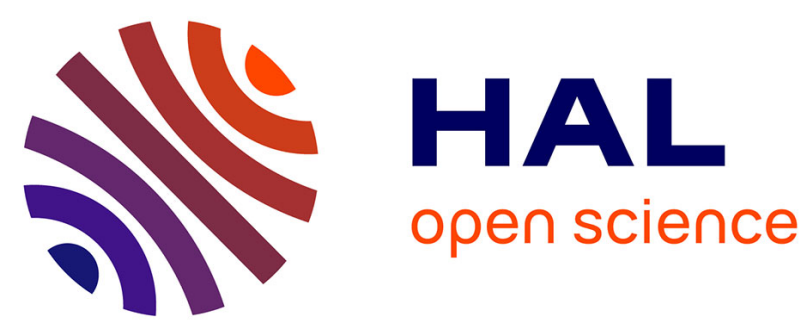

\title{
Comparison between voxel and consistent meso-scale models of woven composites
}

\author{
Aurélien Doitrand, Christian Fagiano, François-Xavier Irisarri, Martin \\ Hirsekorn
}

\section{- To cite this version:}

Aurélien Doitrand, Christian Fagiano, François-Xavier Irisarri, Martin Hirsekorn. Comparison between voxel and consistent meso-scale models of woven composites. Composites Part A: Applied Science and Manufacturing, 2015, 73, pp.143-154. 10.1016/j.compositesa.2015.02.022 . hal-02332834

\section{HAL Id: hal-02332834 \\ https://hal.science/hal-02332834}

Submitted on 9 Apr 2021

HAL is a multi-disciplinary open access archive for the deposit and dissemination of scientific research documents, whether they are published or not. The documents may come from teaching and research institutions in France or abroad, or from public or private research centers.
L'archive ouverte pluridisciplinaire HAL, est destinée au dépôt et à la diffusion de documents scientifiques de niveau recherche, publiés ou non, émanant des établissements d'enseignement et de recherche français ou étrangers, des laboratoires publics ou privés. 
archives-ouvertes

\title{
Comparison between voxel and consistent meso-scale models of woven composites
}

\author{
Aurélien Doitrand, Christian Fagiano, François-Xavier Irisarri, Martin \\ Hirsekorn
}

\section{To cite this version:}

Aurélien Doitrand, Christian Fagiano, François-Xavier Irisarri, Martin Hirsekorn. Comparison between voxel and consistent meso-scale models of woven composites. Composites Part A: Applied Science and Manufacturing, Elsevier, 2015, 73, pp.143-154. 10.1016/j.compositesa.2015.02.022 . hal02332834

\section{HAL Id: hal-02332834 \\ https://hal.archives-ouvertes.fr/hal-02332834}

Submitted on 9 Apr 2021

HAL is a multi-disciplinary open access archive for the deposit and dissemination of scientific research documents, whether they are published or not. The documents may come from teaching and research institutions in France or abroad, or from public or private research centers.
L'archive ouverte pluridisciplinaire HAL, est destinée au dépôt et à la diffusion de documents scientifiques de niveau recherche, publiés ou non, émanant des établissements d'enseignement et de recherche français ou étrangers, des laboratoires publics ou privés. 


\title{
Comparison between voxel and consistent meso-scale models of woven composites
}

\author{
A. Doitrand, C. Fagiano, F.-X. Irisarri, M. Hirsekorn \\ Corresponding author: aurelien.doitrand@onera.fr-01.46.73.46.58 \\ ONERA - The French Aerospace Lab - 92320 Chatillon - France
}

\begin{abstract}
The applicability of voxel meshes to model the mechanical behavior of woven composites at the mesoscopic scale is studied and compared to consistent Finite Element (FE) meshes. The methods are illustrated by mechanically modeling a Representative Unit Cell (RUC) of a composite made of four layers of glass fiber plain weave fabric embedded in an epoxy matrix. Mesh convergence is studied to determine the minimum element size necessary to obtain a correct yarn volume fraction. The comparison between both methods is based on (i) homogenized macroscopic elastic properties, (ii) local stress fields, and (iii) first damage prediction. Even if a good agreement is obtained for the elastic properties, the stress concentrations due to the steplike shape of voxels induce significant differences between both methods in terms of first damage prediction.
\end{abstract}

Keywords: A. Fabrics/textiles, A. Thermosetting resin, C. Finite element analysis, C.

Damage Mechanics

\section{Introduction}

Woven composites are of great interest for industrial applications as they are increasingly used for lightweight applications in aeronautics as well as in the automotive industry. One of the advantages of textile composites is the high flexibility of the reinforcement, which can be draped or directly woven into complex shapes. Therefore, compared to laminated composite structures, fewer assembly operations are needed, which reduces the number of weak points in the structures and production costs.

In an industrial design process, the mechanical properties (in most cases at least the elastic properties and strength) of potentially new materials need to be known. Experimental iden- 
tification is both expensive and time consuming. Moreover, some properties (for composites, e.g., in the out-of-plane direction) are difficult to measure. Therefore, predictive modeling tools are needed to replace some of the experimental tests during the design phase. These modeling tools have to bridge the characteristic scales of composite materials: from the microscopic scale, which is the scale of the constituents (fibers and matrix) to the macroscopic scale, the scale of the structure, at which the material is considered to be homogeneous. An intermediate scale, the so-called mesoscopic scale, is usually defined to take into account the reinforcement architecture. In the case of textile composites, the yarns often consisting of several thousands of fibers are modeled as a homogeneous material. They are interlaced such as to represent the reinforcing fabric architecture and embedded into the matrix material.

The first step in a multi-scale analysis of textile composites [1] is therefore the choice of suitable representative unit cells (RUC) at the different scales. At the meso-scale, the use of yarn shapes and positions that are close to those observed in real composites is extremely important to obtain reliable results [1]. Several tools able to generate geometric models of meso-scale RUC for textile composites exist $[2,3,4]$. However, most of these models imply simplified yarn shapes (constant cross sections) and possible interpenetrations between the yarns, both factors leading to yarn volume fractions lower than those observed experimentally. In order to conserve the overall fiber volume fraction in the composite, very high, sometimes non-physical $[5,6]$ fiber volume fractions have to be used in the yarns. Less idealized geometries can be obtained by explicitly modeling the preforming step of the woven fabric, as it is done, e.g., in $[5,7,8]$. The resulting yarn shapes have varying cross sections as observed in real composites.

With the aid of the meso-scale RUC, the homogenized macroscopic properties of the composite material can be calculated from the properties of the yarns and the matrix at the mescoscopic scale, using a suitable homogenization technique. Analytical methods [9] have the advantage of being computationally very efficient. However, most of them are limited to specific simple reinforcement architectures and all of them only predict linear elastic behavior (non-linear behavior or damage prediction is not considered). Numerical methods, such as Finite Element (FE) modeling are more flexible, as (in principle) any kind of material behavior can be used, and there are no limitations on the geometry, as long as an appropriate FE mesh can be generated. This is in fact one of the main difficulties of this approach, as the 
generation of a periodic mesh at the RUC boundaries (in order to apply periodic boundary conditions) or to impose a conformal mesh at the contact zones between yarns is far from being obvious. The latter problem is often removed artificially by inserting a matrix layer between the yarns in contact $[1,10,11,12]$, which makes the application of automated meshing tools straightforward. If this layer is thin, the element size at the yarn interfaces has to be very small in order to avoid poor quality elements. If it is thick, the fiber volume fraction in the yarns has to be increased significantly above that of the real composite in order to preserve the overall fiber volume fraction [6]. Grail et al. [5] proposed a method for the generation of consistent FE meshes of textile composite RUC with yarns in contact. The contact zones are treated separately and meshed only once, which allows for the generation of conformal meshes without the need of inserting a matrix layer between the yarns. However, this method is complex and its current implementation is not robust enough to ensure automatic mesh generation of any complex 3D reinforcement architecture.

A simpler method, widely used in industrial applications, is voxel meshing [13]. With this method it is quite straightforward to generate meshes of any kind of fabric architecture [14]. It has been used to generate meshes of $2 \mathrm{D}$ woven composites [15] as well as $3 \mathrm{D}$ woven composites with more complex architectures [16]. The voxel method has been successfully used to calculate the homogenized thermo-elastic properties of composites [16]. Several authors also applied voxel meshes to model damage initiation and progression in 3D woven composites $[17,18,19]$. However, spurious stress concentrations are observed at the steplike surfaces of the yarns [15], which are expected to influence damage prediction.

The aim of this work is therefore to evaluate the accuracy and the computational efficiency of the voxel method compared to a consistent FE mesh and to study the range of possible applications during the design phase. The two meshing procedures and a mesh convergence study on the homogenized elastic properties are presented in section 2. The local stress fields are analyzed in section 3 and their influence on damage onset in section 4 . Finally, the stress fields are smoothed in order to reduce the spurious stress concentrations obtained with the voxel meshes (section 5). The whole analysis is illustrated on the RUC of a composite made of a compacted and nested reinforcement of four layers of a glass fiber plain weave fabric embedded in an epoxy matrix. 


\section{Finite element meshing of the Representative Unit Cell}

The first step in FE modeling of woven composites at the meso-scale is the generation of a RUC of the reinforcing fabric. The composite modeled in this work consists of four layers of a glass fiber plain weave fabric embedded in an epoxy matrix. If no particular attention is paid to the position of the fabric layers, they are in general shifted randomly with respect to each other. First, four copies of a unit cell of a single layer of plain weave fabric are generated using the method by Hivet and Boisse [20]. The layers are shifted with respect to each other in the fabric plane, using the shifts determined from experimental observations [21]. The shifted layers are stacked on top of each other. The resulting geometry is repeated periodically in warp and weft direction and then cut to the composite RUC boundaries. A FE mesh is generated of each yarn independently, and the fabric compaction is simulated numerically to obtain the desired final composite thickness, taking into account all the contacts between the yarns [5]. Due to the relative shift between adjacent layers, nesting occurs during compaction. The resulting yarn volume fraction is the same as in the real composite, and the yarn shapes and positions are similar. Although a plain weave is the simplest fabric architecture, the reinforcement geometry already exhibits most of the main difficulties as (i) complex multiple contact zones between the yarns, (ii) irregular yarn shapes with varying sections, (iii) high yarn volume fraction, and (iv) random shifts and nesting between fabric layers. The yarn shapes resulting from the compaction modeling are used in the following to generate FE meshes of the composite RUC made of the deformed reinforcement and the matrix complement. Two methods that ensure conformal meshes at the contact zones between yarns are presented in the following sections 2.1 and 2.2. A mesh convergence study is carried out in section 2.3.

\subsection{Consistent meshing of the Representative Unit Cell}

Grail et al. [5] have developed a method to generate FE meshes of woven composite RUC with complex yarn shapes and multiple contact zones between the yarns. Mesh conformity is ensured as the interfaces between yarns are represented by a single mesh. No artificial matrix layer is needed between yarns, and there are no limitations on yarn shapes. More details concerning this algorithm can be found in [5].

Fagiano et al. [21] have used this method to generate a FE mesh of the RUC studied in this work and to obtain the homogenized macroscopic elastic properties of the material. In a 
first step, the mechanical properties of the yarns are calculated by means of a FE micro-meso homogenization, similar to the method used by Melro et al. [12]. As thermosetting polymer matrices typically used in composites exhibit only weakly nonlinear behavior before failure under tension and strains remain small (see, e.g., [22]), the matrix behavior is supposed to be linear elastic in this study and strain rate dependence is neglected. If the behavior of the composite under compression or shear should be studied, this approximation is no longer valid. The glass fibers are also in a good approximation linear elastic. Both the matrix and the fibers are isotropic materials. The properties used in the following calculations are shown in Table 1. A cylindrical fiber in a hexagonal RUC is used at the micro-scale, which ensures transverse isotropic elastic properties for the yarns. Periodic boundary conditions are applied in all directions. The fiber volume fraction in the yarns is $57 \%$. The homogenized stiffness tensor is calculated from the average stresses in response to the six elementary strains applied consecutively to the RUC. The resulting yarn properties are given in Table 2.

In a second step, the same procedure is applied to the meso-scale FE mesh of the woven composite RUC. However, periodic boundary conditions are only applied in the fabric plane directions of the RUC. The displacement at the out-of-plane faces is left free in order to correctly represent the boundary conditions applied to the composite specimen in a tensile test. The homogenized properties given in Table 2 are used for the yarns and the same properties as at the micro-scale are used for the matrix. The local orientation of the yarn material is calculated separately at each integration point by orthogonal projection of its position on the neutral line of the yarn. The tangent of the neutral line at the projected point defines the axis of transverse isotropy of the yarn material (the fiber direction) at the integration point. The material orientations are assigned automatically using a Python script. The yarn volume fraction in the meso-scale RUC is $82 \%$, resulting in a total fiber volume fraction in the RUC of $47 \%$, which corresponds approximately to yarn volume fraction observed experimentally [21]. The resulting tensile modulus and Poisson's ratio are in good agreement with the experimental observations (Table 3), as well as the strain fields on the surface of the composite. It can be noted that the confidence interval of Poisson's ratio is higher than the confidence interval of Young's modulus. Indeed, the measurement of Poisson's ratio reflects the uncertainty of both longitudinal and transverse strain measurements whereas the measurement of Young's modulus only reflects the former. The damage localization obtained with the consistent 
mesh model has not yet been validated against experimental results. It would require a finer analysis about some aspects affecting the mechanical behavior of the composite e.g. (i) the RUC geometry, (ii) the use of appropriate constitutive laws, etc. However, the principal aim of this paper is the comparison of two FE methods in terms of damage onset localization.

\subsection{Voxel meshing procedure}

The voxel method consists in dividing the composite RUC into a regular 3D grid of voxels. Thus, mesh periodicity at the boundaries of the RUC is ensured automatically. The main difficulty of the method consists in assigning the material properties to each voxel element. In a voxel mesh, the element surfaces do not follow the yarn surfaces but are determined by the regular $3 \mathrm{D}$ grid. Therefore, elements at the yarn surfaces may be shared between several yarns and the matrix. However, it is only possible to assign a single material behavior to each voxel for FE calculations. A solution proposed by Kim and Swan [13] is to define a special material behavior for these elements whose properties are obtained from mixing rules. This implies to calculate as many homogenized material behaviors as there are shared elements in the voxel mesh. Moreover, the definition of the material orientation in elements shared between two yarns of different local fiber orientation is difficult and does not have real physical meaning. Therefore, in most of the studies $[14,15,16]$ the shared elements are assigned to either one yarn or to the matrix. For instance, a possible method is inspired from polycristal's microstructure modeling [24] and consists in growing each part from an initial configuration (e.g., a Voronoi tessellation in the case of polycrystals) until they are in contact. This method as been applied to voxel representations of woven composite RUC by Aboura et al. [23], using the yarn center lines as initial configuration, as it easily allows to assign the voxels to a single material even if there are interpenetrations between the yarns.

Since in this study the geometry is obtained by modeling explicitly the preforming step, there are no interpenetrations between the yarns. Therefore, a simpler method is used to determine to which part a voxel belongs: A voxel is assigned to a yarn or to the matrix depending on its center position. A schematic view of a yarn cross section (black line) is shown in Figure 1, illustrating the voxel assignment to the yarn or the matrix for different voxel sizes. It can be seen that for the same yarn shape, the yarn volume fraction varies

from $58 \%$ (Figure 1 (a)) to $48 \%$ (Figure 1 (b)) depending on the size of the voxel elements. 
Therefore, a mesh convergence study is required to ensure that an accurate yarn volume fraction is used in the FE calculations with voxel meshes. This study is presented in the following section 2.3 .

A general problem of voxel meshes due to the fact that the element boundaries do not follow the yarn surfaces is that artificial contacts between yarns are introduced (see Figure 2). This issue cannot be avoided, but its impact can be reduced by refining the voxel size. A problem of assigning the voxels through their center position is that there may be isolated matrix elements between yarns (see Figures 2 and 3). The center of these elements lies within the matrix but most of their volume belongs to one or several yarns. To overcome this problem, the volume fractions of matrix and yarns are calculated in the shared elements. If in these elements the matrix volume fraction is lower than the volume fraction of at least one of the yarns, it is assigned to the yarn with the bigger volume fraction in the element. Therefore, the elements wrongly assigned to the matrix are assigned to the yarn they mainly belong to (Figure $3)$. Due to the generality of the applied method, this procedure is applicable for any kind of woven fabric architecture. Finally, a third general difficulty of voxel meshes is the representation of the edges of yarns with varying sections where isolated elements can be found (see Figure 3). Such isolated voxels can lead to stress concentrations, see Section 3. This problem is treated and discussed in Section 5. Whatever the voxel size, this phenomenon can be avoided only in yarns with constant cross section and without in-plane waviness, which is not the case in real geometries of compacted fabrics.

\subsection{Mesh convergence study}

The fiber volume fraction has a first order impact on the mechanical behavior of composite materials. For a given fiber volume fraction in the yarns, it is therefore crucial to use the correct yarn volume fraction in the meso-scale RUC. The following mesh convergence study is therefore first carried out on the yarn volume fraction. For a given mechanical behavior, the computational time mostly depends on the number of degrees of freedom of the problem to be solved, which is proportional to the number of nodes in the FE mesh.

One of the most important parameters of the consistent meshing procedure by Grail et

al. [5] is the maximum edge length of the elements. Further parameters include tolerance parameters for the definition of the yarn contact zones and parameters for local refinement 
of the mesh, for example around the yarn interfaces. Details can be found in the paper of Grail et al. [5]. In the present convergence study, only the maximum edge length is varied. The meshing tool tries to mesh the RUC with tetrahedral elements with the maximum edge length. Shorter edges are used only where it is required to accurately reproduce the geometry and close the meshes. The shorter the maximum edge length, the higher the number of nodes in the FE mesh, but the better the curvatures of the geometry are approximated. The yarn volume fraction of the consistent meshes remains constant within about $0.03 \%$ from a maximum edge length of $0.25 \mathrm{~mm}$ downwards (see Figure 4). If 10 node quadratic elements are used, this corresponds to a FE mesh with 259000 nodes and a yarn volume fraction of 82.18\%. The mesh is shown in Figure 5.

The number of nodes in a voxel mesh made of linear hexahedral elements is given by

$$
n_{\text {nodes }}=\left(n_{x}+1\right)\left(n_{y}+1\right)\left(n_{z}+1\right)
$$

where $n_{x}$ is the number of voxels in direction of the warp yarns, $n_{y}$ in direction of the weft yarns, and $n_{z}$ through the thickness of the RUC. In most published works involving voxel meshes of woven composites cubic voxels are used $[14,15,16]$. The mesh size thus depends only on one parameter: the length of the voxel edge. For the present RUC, 2000000 nodes are needed to obtain a difference of less than $0.4 \%$ between the yarn volume fractions of the converged consistent mesh and that of the voxel mesh, if cubic voxels are used.

An alternative is to use hexahedral voxels with different edge lengths in the three directions. Since the yarn width in the studied fabric is the same in warp and weft direction, we choose the same voxel size in the fabric plane, i.e.,

$$
n_{y}=\left[\frac{L_{y}}{L_{x}}\right] n_{x}
$$

where $L_{x}$ and $L_{y}$ are respectively the RUC length in warp and weft direction. The number of voxels in the thickness direction is chosen independently, leading to flatter voxels.

The voxel size in thickness direction and hence the number of nodes needed to obtain a relative difference lower than $0.25 \%$ between the yarn volume fractions of the consistent mesh and those of the voxel meshes depends on the number of voxels in the plane $n_{x}$. The evolution of the yarn volume fractions with the number of nodes is shown in Figure 4 for three different values of $n_{x}$. For $n_{x}=20$, convergence is reached for $n_{z}=150$, which corresponds to a 
voxel mesh with 72900 nodes. Note that in contrast to the consistent mesh, linear elements are used in the voxel meshes, because the use of quadratic elements does not improve the representation of the steplike geometry, but only increase the number of nodes and hence the computational costs. If the consistent mesh shown in Figure 5 was made of linear tetrahedral elements (which would be sufficient if the objective was only to get a correct volume fraction as the yarn volume fraction does not change if linear or quadratic elements are used), it would only contain 34100 nodes. Therefore, using a consistent mesh with tetrahedral elements, fewer nodes are needed to correctly represent the RUC geometry than with a voxel mesh consisting of hexahedral elements..

However, such a low number of voxels in the plane insufficiently describes the geometrical characteristics of the RUC and thus alters the local stress fields significantly, as will be shown in the following section. Therefore, a finer discretization is required if the mesh should not only be used to compute homogenized elastic properties. The converged voxel mesh with $n_{x}=70$ and $n_{z}=90$ is shown in Figure 6. This mesh contains 504000 nodes. Thus, even for high $n_{x}$ the use of hexahedral voxels drastically reduces the number of nodes required to achieve convergence of the yarn volume fraction compared to cubic voxels.

The homogenized elastic properties have been calculated using the consistent mesh and the different voxel meshes and following the methods described in section 2.1. As both the voxel meshes and the consistent mesh are periodic, the application of periodic boundary conditions is straightforward. It has been found that the homogenized elastic properties are mainly influenced by the yarn volume fraction in the RUC and the distribution of the fiber orientations. The exact yarn shapes are less important to the averaged elastic behavior. To obtain a correct fiber orientation distribution, the yarns have to be sufficiently well discretized along the fiber direction. As in a plain weave, the yarn path is similar to a sinusoidal curve, $n_{x}=20$ voxels in the plane are sufficient. Therefore, for voxel meshes with converged yarn volume fractions, the homogenized elastic properties are also converged with a similar precision. The results for the mesh shown in Figure 6 are given in Table 4 compared with the values obtained with the consistent mesh. This study shows that voxel meshes are well suited to calculate the homogenized elastic properties of textile composites. The computational costs are similar to those that can be reached with consistent FE meshes. 


\section{Local stress fields}

Local stress concentrations only have a minor influence on the homogenized elastic properties, as they are averaged out over the RUC. Therefore, the steplike structure of the yarn surfaces in the voxel meshes does not have a major impact, as long as the yarn volume fraction and the fiber orientation distribution are correctly represented. In this section, the local stress fields are compared between the voxel and the consistent meshes, in order to asses the viability of the voxel method for the prediction of phenomena that depend on local stress concentrations, such as damage onset. Since the first damage observed in textile composites under tension is usually transverse yarn cracking $[1,25,26]$, a weft yarn of one of the internal layers is chosen to study the stress fields (the red yarn in Figure 7). A tensile load is imposed in the warp direction. The average stress in the other directions of the RUC is zero.

The in-plane transverse stress fields in the selected yarn obtained with different voxel meshes are shown in Figure 8. Four different voxel numbers $\left(n_{x}\right)$ are chosen in the in-plane direction. The number of voxels through the thickness $n_{z}$ is fixed as a result of the convergence study presented in section 2.3. It can be clearly seen that for $n_{x}=20$ the voxels are too big to reproduce the geometric features of the meso-scale RUC. As a results, the stress concentration zones observed with finer voxel meshes are dilated and their shape is not accurately reproduced. For $n_{x}=40$ the characteristic shapes of the stress fields observed with the two finer meshes start to appear, but the approximation of their shape is still very rough. For the two finest meshes, the general shape of the stress fields are very similar, with the exception of the stress concentrations due to the steplike yarn surface.

These stress concentrations are observed in all voxel meshes, regardless the voxel size. They are even more important in the finer meshes, as for the very coarse mesh the yarn shape is too much simplified. This is reflected by the maximum local tensile stress, which increases with decreasing voxel size. The impact on the minimum local stress is less clear, as it first decreases with the voxel size, which is probably due to the increasingly more accurate representation of the yarn shape, but then increases again for the finest mesh.

The in-plane transverse stress fields in the selected yarn obtained with different consistent meshes are shown in Figure 9. Four different mesh sizes (0.6, 0.25, 0.18 and $0.1 \mathrm{~mm})$ are chosen. It can be clearly seen that a mesh size of $0.6 \mathrm{~mm}$ is too coarse to well describe the 
stress field. For the three finest meshes, the obtained elastic properties as well as the shape of the stress fields are very similar. Hence, if the mesh size is small enough, the stress field does not vary locally contrary to the voxel case.

In terms of local stress fields, tetrahedral elements are less precise than hexahedral elements [27]. However, it is really difficult to generate a consistent mesh with hexahedral elements, due to the complexity of the geometry of woven composites. Tetrahedral elements have to be used in order to obtain such a mesh. Quadratic instead of linear elements are chosen in order to better take into account stress gradients that may occur through the yarn thickness.

The consistent mesh with a converged yarn volume fraction shown in Figure 5 only has two elements in the direction of the yarn thickness, which is the minimum imposed by the algorithm of Grail et al. [5]. This is sufficient to obtain a good convergence in yarn volume fraction and homogenized elastic properties, but usually insufficient to take into account the stress gradients that may occur through the yarn thickness. In the following, we therefore use a refined mesh with four elements through the yarn thickness and a total of 845000 nodes, which should be sufficient because quadratic elements are used in the consistent mesh. The obtained stress fields are shown in Figure 10 for both consistent meshes and for the voxel mesh with $n_{x}=70$. There is no significant difference between the stress fields in the two consistent meshes. Nevertheless, the refined mesh will be used in the following analysis in order to better reproduce the stress gradients in the through-thickness direction. In all converged voxel meshes, the number of elements through the yarn thickness is more than twice of that in the consistent meshes, which means that stress gradients should be at least equally well be taken into account.

A comparison between the stress fields on the whole yarn surface of the voxel mesh with $n_{x}=70$ and the refined consistent mesh is shown in Figure 11. The zones where this yarn is in contact with other yarns are surrounded by black lines. The minimum and maximum local stresses observed with these two meshes are summarized in Table 5. Both values are quite similar. The voxel mesh with $n_{x}=70$ gives the same overall shapes of the stress fields as the mesh with $n_{x}=100$. However, mesh dependent stress oscillations are obtained with both meshes, so that there is no advantage in using the finer mesh. Therefore, in order to perform a quantitative comparison between voxel and consistent mesh results, the mesh with $n_{x}=70$ will be used in the following comparisons. 
The general shape of the stress fields shown in Figure 11 are quite similar, with the exception of the local oscillations between stress extrema induced by the steplike yarn surfaces in the voxel mesh. The stress oscillations are caused by the inaccurate representation of the yarn surface shape in the voxel mesh, and are therefore a geometric artifact not due to a physical mechanism. These oscillations are more important at interfaces between yarns than at interfaces between the yarn and the matrix. Even though the overall maxima and minima of local stress are quite similar (Table 5), differences of more than $30 \%$ are reached in the zones with stress oscillations. A zoom on a region with stress oscillations is shown in Figure 10. In the same region, the stress field obtained with the consistent meshes is smooth without oscillations.

\section{Damage onset}

One of the most important criteria in a design process of composite structures is the level of damage onset, which is highly influenced by the local stress distributions. The differences highlighted in Figures 10 and 11 are therefore expected to significantly impact the prediction of the first damage. In order to estimate numerically damage onset in the yarns, a suitable damage criterion has to be used. The yarns are made of fibers embedded in matrix, which are locally almost parallel to each other, similar to a unidirectional (UD) ply. We therefore use a damage criterion developed for UD plies in a composite laminate $[28,29]$ to locate the first damage in the yarns. In this model, three different damage modes are taken into account: (1) damage in the direction of the fibers dominated by fiber breaking and matrix dominated damage in (2) the transverse in-plane direction and (3) the out-of-plane direction. For each mode, tensile and compressive damage are distinguished, as the physical mechanisms are not the same. The criteria for the different modes are given in Table 6 .

The lower index in the failure criteria $f_{i}^{ \pm}$indicates the damage mode, and the upper index distinguishes between tensile $(+)$ and compressive $(-)$ damage. $X_{t}$ and $X_{c}$ are the tensile and compressive ply strength in direction of the fibers, which are determined by tensile and compressive tests on UD plies. $Y_{t}$ and $Y_{c}$ are the transverse tensile and compressive strengths. $S_{12}^{f}$ and $S_{13}^{f}$ are called the effective shear strengths. They are higher than the real shear strengths $S_{12}^{R}$ and $S_{13}^{R}$ of the ply such that damage onset is predicted correctly in a matrix-dominated mode (2 or 3 ) for pure shear loading, and not in the compressive 
fiber failure mode [29]. $p_{12}, p_{13}$ are shape parameters that take into account the coupling between compressive and shear stresses. These parameters can be directly determined from the transverse compressive ply strength $Y_{c}$ [29]. For the polymer matrix, a failure criterion proposed by Chaboche et al. [30] for quasi-brittle materials is used (last equation in Table 6 ). Failure is driven by the principal tensile stresses $\left\langle\sigma_{I}\right\rangle,\left\langle\sigma_{I I}\right\rangle$ and $\left\langle\sigma_{I I I}\right\rangle$. $X$ is the matrix strength under uniaxial tension. Triaxiality is accounted for through the exponent $p$, which is set to 1 in this study.

Damage occurs when any of the different criteria becomes larger than 1 . The integration point where this occurs first in the FE calculation gives the location of damage onset. The local damage mode is given by the criterion $f$ that is maximum at the integration point.

Experimentally, the first damage mechanism observed in woven composites is transverse cracking in yarns that are orthogonal to the loading direction. The next damage mechanisms are (i) yarn/yarn or yarn/matrix decohesions at the crack tips, (ii) matrix cracking in the matrix pockets and (iii) fiber fracture leading to the failure of the material. A more detailed description of the damage mechanisms can be found in [21] and [26]. At damage onset, only the transverse yarn cracking mode of the failure criterion is active $\left(\max (f)=f_{2}^{+}\right)$, which agrees with the experimental observations. Therefore, the results presented in the following only concern the transverse yarn cracking mode of the failure criterion.

The damage onset locations obtained with the different voxel meshes presented in Figure 8 are shown in Figure 12. Damage onset prediction is clearly influenced by the stress concentrations that are observed for all the meshes. The damage onset locations obtained for the reference weft yarn with the voxel and consistent mesh are shown in Figure 13. In order to identify a damage zone rather than a single integration point, the applied load has been fixed such that the maximum failure criterion $\max (f)$ is 1.1 . This value is obtained for an average tensile stress of $72.6 \mathrm{MPa}$ in the warp direction. In Figure 13 all areas associated to integration points where $\max (f)$ lies between 1 and 1.1 are marked in red.

For both meshes, damage onset is observed at the locations of maximum transverse tensile stress in the yarns (see Figure 11). Some damage zones are similar for both methods, but additional damaged locations are predicted with the voxel method at the locations of local stress concentrations that are due to the steplike yarn surface, which are not present in the consistent mesh. In particular, the first damage predicted by the voxel method is located in 
such a zone. However, an overall maximum failure criterion of 1 is reached for both methods at similar loads, with a difference of only $0.6 \%$ between them.

\section{Stress field smoothing}

The differences between the consistent and the voxel meshes in the prediction of damage zones is due to the oscillations between stress maxima and minima at the steplike yarn surface in the voxel mesh. These oscillations can be reduced by smoothing the stress field. This is done by calculating the stress at each integration point as an interpolation of the non-smoothed values at the surrounding integration points using a least squares method [31]. The amount of smoothing depends on the number of surrounding integration points that are taken into account. Usually, all integration points are included that belong to the elements that share a common node with the element containing the integration point where the smoothed field is calculated. In this case, at each integration point, the stress field is smoothed over 216 integration points. If the integration points of second nearest neighbor elements are also included, the number rises to 1000, for the third nearest neighbors it becomes 2744 .

The stress concentration smoothing leads to two configurations: (i) if the stress concentration is localized in only one element of the zone of interest, see Figure 14 (1a), the smoothing technique is sufficient to reduce significantly the stress concentration in the isolated element, see Figure 14 (1b); (ii) if several elements are subject to stress concentrations in the same zone, see Figure 14 (2a), the smoothing method may not be sufficient to completely reduce this effect, see Figure 14 (2b). In this case, a damage zone may be detected erroneously.

The resulting smoothed stress fields are shown in Figure 15.The oscillations observed in Figure 11 are no longer present. However, the stress maxima are decreasing and the stress minima are increasing with the number of integration points included in the smoothing process. This has an impact on the applied load level, for which damage onset is predicted. For example, for an average stress of $72.6 \mathrm{MPa}$ in the warp direction, the maximum failure criterion reaches only $\max (f)=0.97$ after smoothing over 216 integration points instead of 1.1 if the non-smoothed stress fields are used. As a consequence, a $13 \%$ higher average stress $(82.5 \mathrm{MPa})$ is needed to reach the same maximum failure criterion $\max (f)=1.1$ as with the consistent mesh. 
The smoothing procedure also has an influence on the predicted location of damage. In Figure 16 a comparison is shown between the results obtained with the non-smoothed and the smoothed stress fields of the voxel meshes and with the consistent mesh. The zones attributed to integration points with $f$ between 1 and 1.1 are highlighted in red. The load applied to each mesh is chosen such that the maximum local value of $f$ is 1.1. The Figure shows that the smoothing removes some damage zones and the remaining zones spread out slightly. This has the result that most of the damage zones caused by the stress oscillations at the steplike yarn surfaces are removed, but also some relatively localized damage zones predicted with the consistent mesh. Moreover, some damage zones not predicted with the consistent mesh are not removed after smoothing. Further smoothing including the second and third nearest neighbor elements has no major impact on the predicted damage zones. As can be seen from Figure 15, the zones of maximum stress and their locations remain almost the same. They only spread out slightly more. The same effect is observed on the damage zones.

Smoothing the stress fields is therefore only an insufficient solution to correct the stress oscillations caused by the steplike approximation of the yarn shapes. Since the maximum local stress depends on the level of smoothing, it is not appropriate to predict the absolute value of the damage threshold. The voxel size also has an influence on the maximum local stress, which may result in a mutual compensation of mesh refinement and stronger smoothing. However, this does not mean that convergence is reached.

\section{Conclusion}

The applicability of the voxel method for mesoscale modeling of woven composites has been studied using a RUC of a polymer matrix composite with a compacted and nested reinforcement made of four layers of plain weave fabric. In comparison with the results obtained with a consistent mesh, whose element faces follow the yarn surfaces, the voxel mesh yields stress oscillations at the yarn surfaces. These are due to a less precise approximation of the geometry of the reinforcement by the voxel mesh, which cannot represent smooth curved surfaces. These oscillations average out, when the homogenized elastic properties are calculated. For this reason, it is sufficient to choose the voxel size small enough to obtain an accurate value for the yarn volume fraction. If hexahedral rather than cubic elements are used for the voxel mesh, this is achieved for similar node numbers in the voxel and the consistent 
mesh. Therefore, the voxel method is a well suited and flexible method to determine the average elastic behavior of composite RUC with highly complex reinforcement geometries.

However, if phenomena depending on local stress concentrations, such as damage onset, are simulated, the difference between voxel and consistent meshes becomes significant. Using a voxel mesh, damage is not only predicted in zones of stress concentration due to the geometry of the reinforcement, but also at the stress oscillations at the steplike yarn surfaces. The effect is particularly strong at the interfaces between two crossing yarns. The damage location is thus incorrectly predicted in most cases. The damage threshold is also influenced, since the stress concentrations at the steplike yarn surface depend on the voxel size. In fact, in these zones, local differences of more than $30 \%$ are observed between the voxel and the consistent mesh. Moreover, these stress oscillations are present regardless of the mesh size. Therefore, the choice of a good mesh discretization to generate appropriate stress fields or to perform damage analyses is not obvious.

The stress oscillations can be reduced by smoothing the stress fields. However, the smoothing does not remove all damage zones that are predicted only due to the stress concentrations at the steplike yarn surfaces. Moreover, some damage zones that are in agreement with those predicted with the consistent mesh, are also removed. Even after smoothing, the prediction of the damage location is thus not reliable and depends on the voxel size. The maximum local stress, which determines the damage threshold, depends both on the level of smoothing and on the voxel size. Both effects may compensate each other if the voxel mesh is refined and the smoothing level increased. This makes the prediction of the damage threshold strongly dependent on the parameters of the procedure.

The main conclusion of this article is that it has been found that under some conditions the voxel method is well suited to calculate the homogenized elastic properties of composites, but it has to be used with caution when applied to the simulation of phenomena that depend on local stress concentrations, such as damage onset and propagation. Therefore, the consistent mesh method will be used for further work covering an experimental validation of the FE model in terms of damage localization. 


\section{References}

[1] Lomov SV, Ivanov DS, Verpoest I, Zako M, Kurashiki T, Nakai H, Hirosawa S. MesoFE modelling of textile composites: Road map, data flow and algorithms. Compos Sci Technol 2007;67(9):1870-91.

[2] Lomov SV, Gasakov AV, Huysmans G, Prodromou A, Verpoest I. Textile geometry preprocessor for meso-mechanical models of woven composites. Compos Sci Technol 2000;60(11):2083-95.

[3] Sherburn M. Geometric and Mechanical Modelling of Textiles. Ph.D. thesis, The University of Nottingham; 2007.

[4] Couegnat G. Approche multiéchelle du comportement mécanique de matériaux composites à renfort tissé. Ph.D. thesis, Universit de Bordeaux 1; 2008.

[5] Grail G, Hirsekorn M, Wendling A, Hivet G, Hambli R. Consistent Finite Element mesh generation for meso-scale modeling of textile composites with preformed and compacted reinforcements. Compos Part A: Appl Sci Manuf 2013;55:143-51.

[6] Ivanov DS, Baudry F, Van Den Broucke B, Lomov SV, Xie H, Verpoest I. Failure analysis of triaxial braided composite. Compos Sci Technol 2009;69(9):1372-80.

[7] Hua L, Sherburn M, Crookston J, Long A, Clifford M, Jones I. Finite element modelling of fabric compression. Model Simul Mater Sci Eng 2008;16(3).

[8] Nguyen QT, Vidal-Sallé E, Boisse P, Park CH, Saouab A, Bréard J, Hivet G. Mesoscopic scale analyses of textile composite reinforcement compaction. Compos Part B: Eng 2012;44(1):231-41.

[9] Hallal A, Younes R, Fardoun F. Review and comparative study of analytical modeling for the elastic properties of textile composites Original Research Article. Compos Part B: Eng 2013;50:22-31.

[10] Stig F, Hallstrom S. A modelling framework for composites containing 3D reinforcement. Compos Struct 2012;94(9):2895-901. 
[11] De Carvalho NV, Pinho ST, Robinson P. Numerical modelling of woven composites: biaxial loading. Compos Part A: Appl Sci Manuf 2012;43(8):1326-37.

[12] Melro AR, Camanho P, Andrade Pires FM, Pinho ST. Numerical simulation of the nonlinear deformation of 5-harness satin weaves. Comp Mater Sci 2012;61(0):116-126.

[13] Kim HJ, Swan CC. Voxel-based meshing and unit-cell analysis of textile composites. Int. J. Numer. Meth. Eng 2003;56:977-1006.

[14] Potter E, Pinho ST, Robinson P, Iannucci L, McMillan AJ. Mesh generation and geometrical modeling of $3 \mathrm{D}$ woven composites with variable tow cross-sections. Comput Mater Sci 2012;51(1):103-11.

[15] De Carvalho NV, Pinho ST, Robinson P. Reducing the domain in the mechanical analysis of periodic structures, with application to woven composites. Compos Sci Technol 2011;71(7):969-79.

[16] Schneider J. A Meso-Voxel model of an interlock woven composite. In: Proceedings of ICCM-17 Conference. Edinburgh, July, 2009.

[17] Koumpias AS, Tserpes KI, Pantelakis S. Progressive damage modelling of 3D fully interlaced woven composite materials. Fatigue and Fracture of Engineering Materials and Structures 2014;37(7):696-706.

[18] Green SD, Matveev MY, Long AC, Ivanov D, Hallett SR. Mechanical modelling of 3D woven composites considering realistic unit cell geometry. Compos Struct 2014;118:28493.

[19] Zeng X, Brown LP, Endruweit A, Matveev M, Long AC. Geometrical modelling of 3D woven reinforcements for polymer composites: Prediction of fabric permeability and composite mechanical properties. Compos Part A 2014;56:150-60.

[20] Hivet G, Boisse P. Consistent 3D geometrical model of fabric elementary cell. Application to a meshing preprocessor for 3D finite element analysis. Finite Elements Anal Des 2005;42(1):25-49. 
[21] Fagiano C, Hirsekorn M, Grail G, Chiaruttini V, Lapeyronnie P. Characterization and modeling of damage at the mesoscale of woven polymer matrix composites. In: Proceedings of ICCM-19, Montreal, July, 2013.

[22] Kästner M, Blobel S, Obst M, Thielsch K, Ulbricht V. Experimental characterization of the viscoplastic material behaviour of thermosets and thermoplastics. Applied Mechanics and Materials 2010;24-25:195-200.

[23] Aboura Z, Hello G, Kebir H, Tiar M-A, Benzeggagh M-L. Finite element modeling of mechanical behavior of 3D composite materials. In: Proceedings of ECCM-15 Conference. Venice, June, 2012.

[24] Svyetlichnyy D-S. Modelling of the microstructure: From classical cellular automata approach to the frontal one. Computational Materials Science 2010;50(1):92-97.

[25] John S, Herszberg I, Coman F. Longitudinal and transverse damage taxonomy in woven composite components. Compos Part B Eng 2001;32(8),659-68.

[26] Daggumati S, De Baere I, Van Paepegem W, Degrieck J, Xu J, Lomov SV, Verpoest I. Local damage in a 5-harness satin weave composite under static tension: Part I Experimental analysis. Compos Sci Technol 2010;70(13),1926-33.

[27] Fagiano C, Genet M, Baranger E, Ladevèze P. Computational geometrical and mechanical modeling of woven ceramic composites at the mesoscale. Compos Struct 2014;112:14656.

[28] Laurin F, Carrère N, Maire J-F. A multiscale progressive failure approach for composite laminates based on thermodynamical viscoelastic and damage models. Compos. Part A 2007;38(1):198-209.

[29] Charrier JS. Développement de méthodologies dédiées à l'analyse robuste de la tenue de structures composites sous chargements complexes tridimensionnels. Ph.D. thesis, Onera and ENSAM, 2013.

[30] Chaboche JL, Lesne PM, Maire J-F. Phenomenological damage mechanics of brittle materials with description of unilateral damage effects. In: Bažant ZP, Bittnar Z, Jirásek 
M, Mazars J (Eds.), Fracture and Damage in Quasi-Brittle Structures, E \& FN SPON, London (1994) 75-84.

[31] Feld-Payet S. Amorçage et propagation de fissures dans les milieux non locaux. Ph.D. thesis, ENSMP, 2010. 


\begin{tabular}{|c|c|c|c|c|c|}
\hline Matrix & $E[\mathrm{GPa}]$ & $\nu$ & Fibres & $E[\mathrm{GPa}]$ & $\nu$ \\
\hline LY564 & 3.2 & 0.35 & E-glass & 72 & 0.3 \\
\hline
\end{tabular}

Table 1: Microscopic properties of the constituents: E-glass fiber and epoxy matrix.

\begin{tabular}{|c|c|c|c|c|c|}
\hline Matrix & $E[\mathrm{GPa}]$ & $\nu$ & & & \\
\hline LY564 & 3.2 & 0.35 & & & \\
\hline Yarns & $E_{l}[\mathrm{GPa}]$ & $E_{t}[\mathrm{GPa}]$ & $\nu_{l t}$ & $\nu_{t t}$ & $G_{l t}[\mathrm{GPa}]$ \\
\hline & 42.2 & 10.2 & 0.319 & 0.419 & 7.6 \\
\hline
\end{tabular}

Table 2: Mesoscopic properties of the constituents obtained from micro-meso homogenization. 


\begin{tabular}{|c|c|c|}
\hline Method & $E_{11}[\mathrm{GPa}]$ & $\nu_{12}$ \\
\hline Finite element & 20.94 & 0.132 \\
\hline Tests & $21.31 \pm 0.6$ & $0.148 \pm 0.027$ \\
\hline
\end{tabular}

Table 3: Macroscopic elastic properties of the four-layer plain architecture obtained numerically (FE) and experimentally [21].

\begin{tabular}{|c|c|c|c|c|c|}
\hline Properties & $E_{11}[\mathrm{GPa}]$ & $E_{22}[\mathrm{GPa}]$ & $\nu_{12}$ & $\nu_{23}$ & $G_{12}[\mathrm{GPa}]$ \\
\hline Consistent mesh & 20.94 & 22.39 & 0.132 & 0.413 & 6.47 \\
\hline Voxel mesh & 20.90 & 22.32 & 0.133 & 0.412 & 6.46 \\
\hline Difference (\%) & 0.2 & 0.31 & 0.29 & 0.23 & 0.18 \\
\hline
\end{tabular}

Table 4: Macroscopic elastic properties of the constituents obtained from meso-macro homogenization using consistent and voxel meshes, and the relative difference between both methods. 


\begin{tabular}{|c|c|c|}
\hline Properties & $\sigma_{\min }[\mathrm{MPa}]$ & $\sigma_{\max }[\mathrm{MPa}]$ \\
\hline Consistent mesh & 18.82 & 48.82 \\
\hline Voxel mesh & 18.14 & 47.95 \\
\hline Difference $(\%)$ & 3.7 & 1.8 \\
\hline
\end{tabular}

Table 5: Maximum and minimum local stress in the studied weft yarn obtained with consistent and voxel meshes.

\begin{tabular}{|c|c|}
\hline 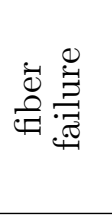 & $\begin{array}{l}f_{1}^{+}=\left(\frac{<\sigma_{11}>}{X_{t}}\right)^{2} \\
f_{1}^{-}=\left(\frac{\leq-\sigma_{11}>}{X_{c}}\right)^{2}+\left(\frac{\tau_{12}}{S_{12}^{f}\left(1-p_{12} \sigma_{22}\right)}\right)^{2}+\left(\frac{\tau_{13}}{S_{13}^{f}\left(1-p_{13} \sigma_{33}\right)}\right)^{2} \\
<x>=x \text { if } x>0,<x>=0 \text { otherwise }\end{array}$ \\
\hline 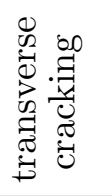 & $\begin{array}{l}f_{2}^{+}=\left(\frac{<\sigma_{22}>}{Y_{t}}\right)^{2}+\left(\frac{\tau_{12}}{S_{12}^{R}\left(1-p_{12} \sigma_{22}\right)}\right)^{2}+\left(\frac{\tau_{23}}{S_{23}^{R}\left(1-p_{23}\left(\sigma_{22}+\sigma_{33}\right)\right)}\right)^{2} \\
f_{2}^{-}=\left(\frac{<-\sigma_{22}>}{Y_{c}}\right)^{2}+\left(\frac{\tau_{12}}{S_{12}^{R}\left(1-p_{12} \sigma_{22}\right)}\right)^{2}+\left(\frac{\tau_{23}}{S_{23}^{R}\left(1-p_{23}\left(\sigma_{22}+\sigma_{33}\right)\right)}\right)^{2}\end{array}$ \\
\hline 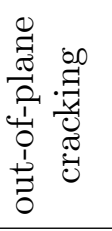 & $\begin{array}{l}f_{3}^{+}=\left(\frac{<\sigma_{33}>}{Y_{t}}\right)^{2}+\left(\frac{\tau_{13}}{S_{13}^{R}\left(1-p_{13} \sigma_{33}\right)}\right)^{2}+\left(\frac{\tau_{23}}{S_{23}^{R}\left(1-p_{23}\left(\sigma_{22}+\sigma_{33}\right)\right)}\right)^{2} \\
f_{3}^{-}=\left(\frac{\leq-\sigma_{33}>}{Y_{c}}\right)^{2}+\left(\frac{\tau_{13}}{S_{13}^{R}\left(1-p_{13} \sigma_{33}\right)}\right)^{2}+\left(\frac{\tau_{23}}{S_{23}^{R}\left(1-p_{23}\left(\sigma_{22}+\sigma_{33}\right)\right)}\right)^{2}\end{array}$ \\
\hline 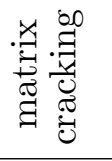 & $f_{\text {matrix }}=\frac{\left(<\sigma_{I}>^{p}+<\sigma_{I I}>^{p}+<\sigma_{I I I}>^{p}\right)^{1 / p}}{X}$ \\
\hline
\end{tabular}

Table 6: Failure criteria for the yarns (first three rows) and the matrix used in the damage predictions. 

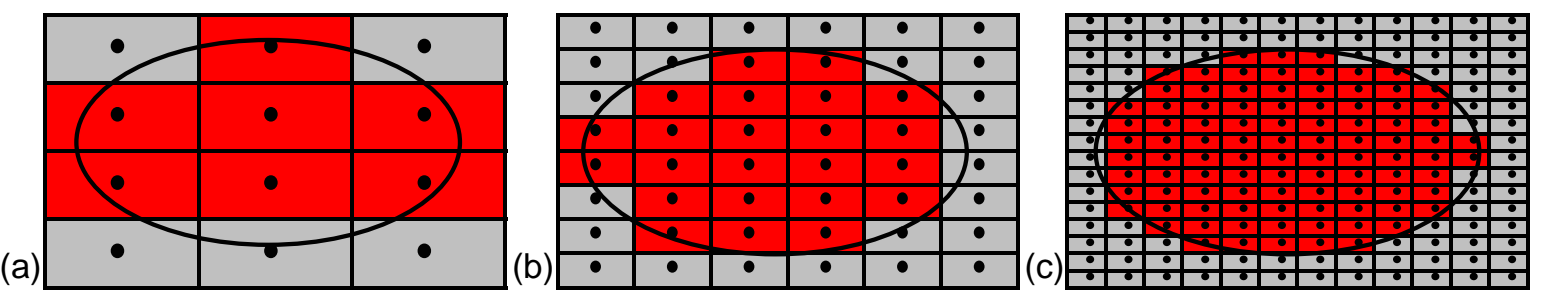

Figure 1: Schematic view of a yarn cross section. Elements are assigned to a yarn (in red) or to the matrix according to the position of their centers. The yarn volume fraction depends on the voxel size and is equal to (a) 0.58 (b) 0.48 , and (c) 0.49 .

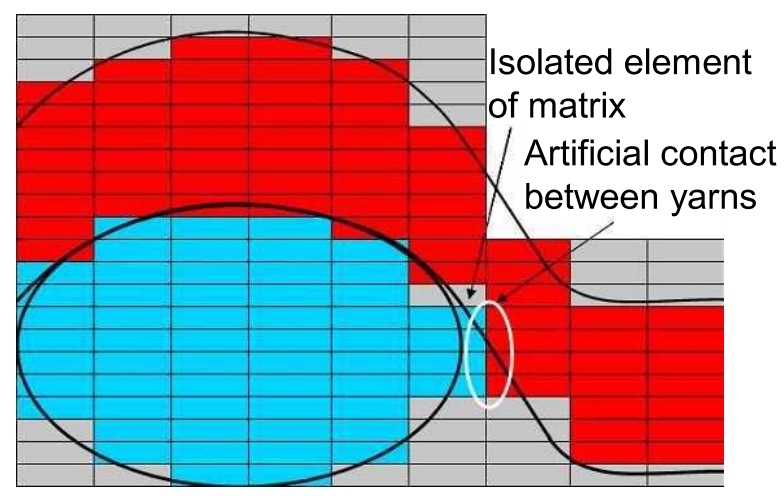

Figure 2: Illustation of isolated matrix elements and artificial contacts between yarns generated by assigning voxels by their center position. Yarns are represented in red and blue, the matrix in grey. 

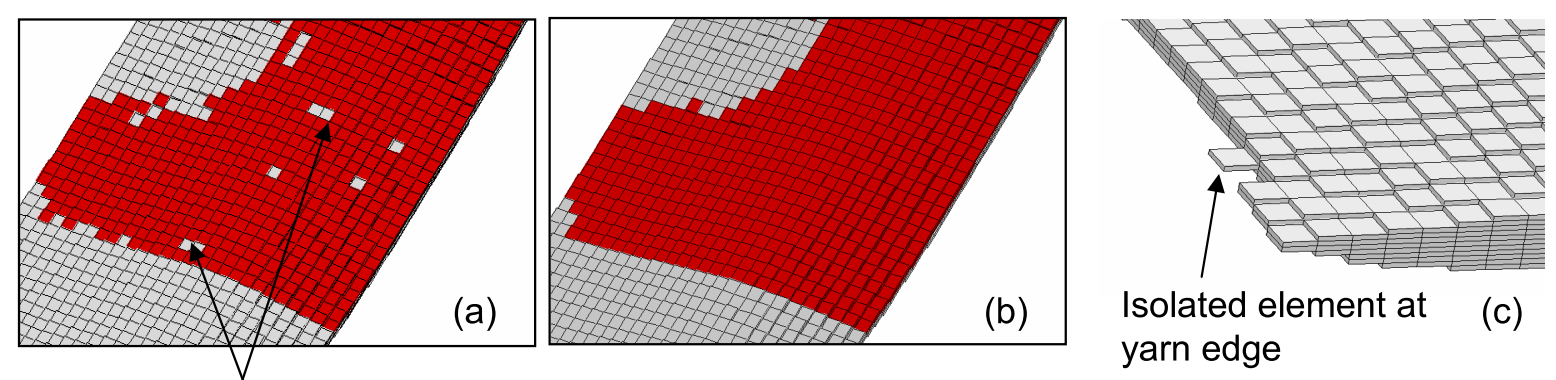

Isolated elements of matrix

Figure 3: The interface between two yarns is represented in red before (a) and after (b) reassigning isolated matrix elements. (c) shows an example for isolated elements at yarn edges.

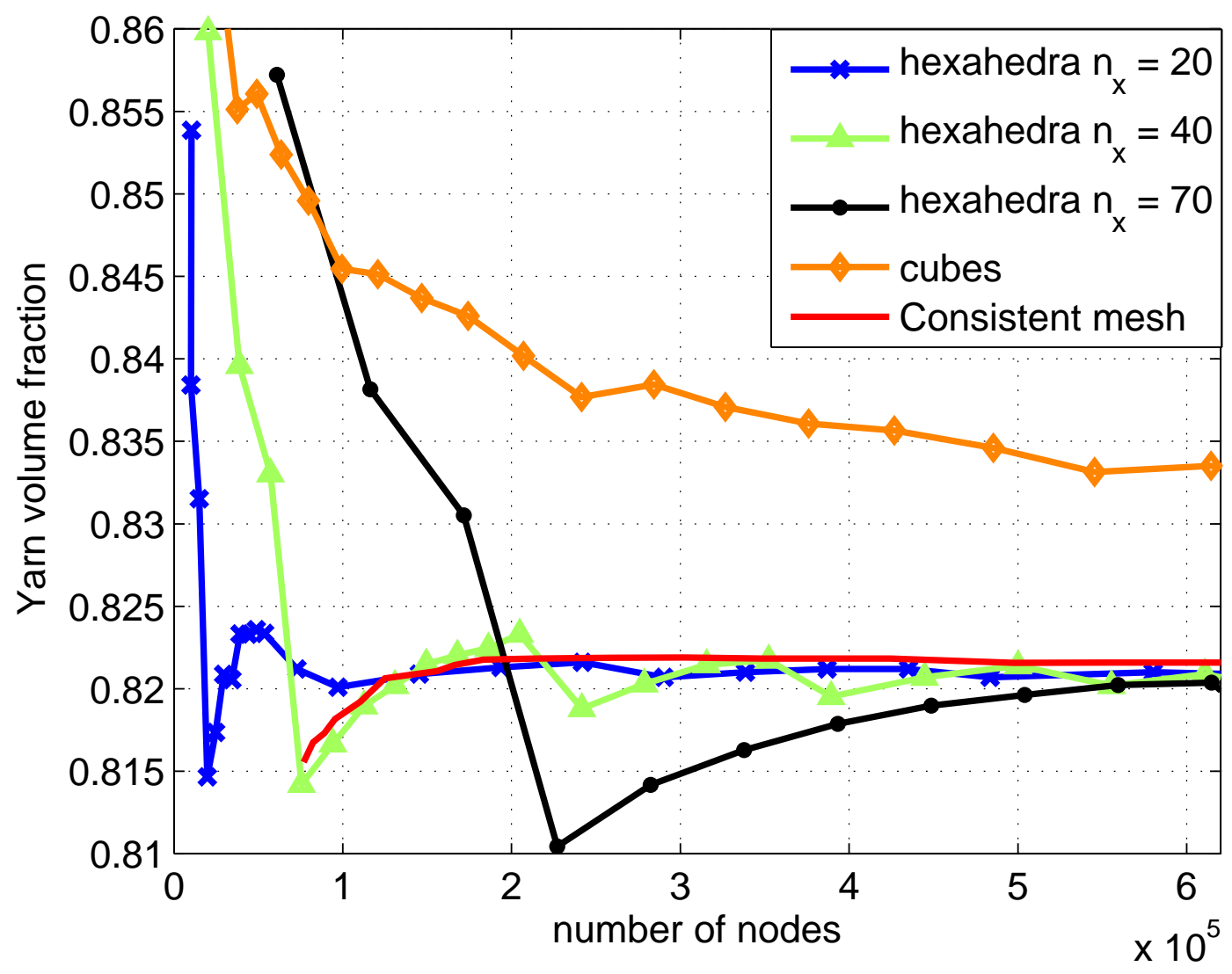

Figure 4: Evolution of the yarn volume fraction with the number of nodes for consistent meshes and for voxel meshes with different voxel sizes and shapes. 


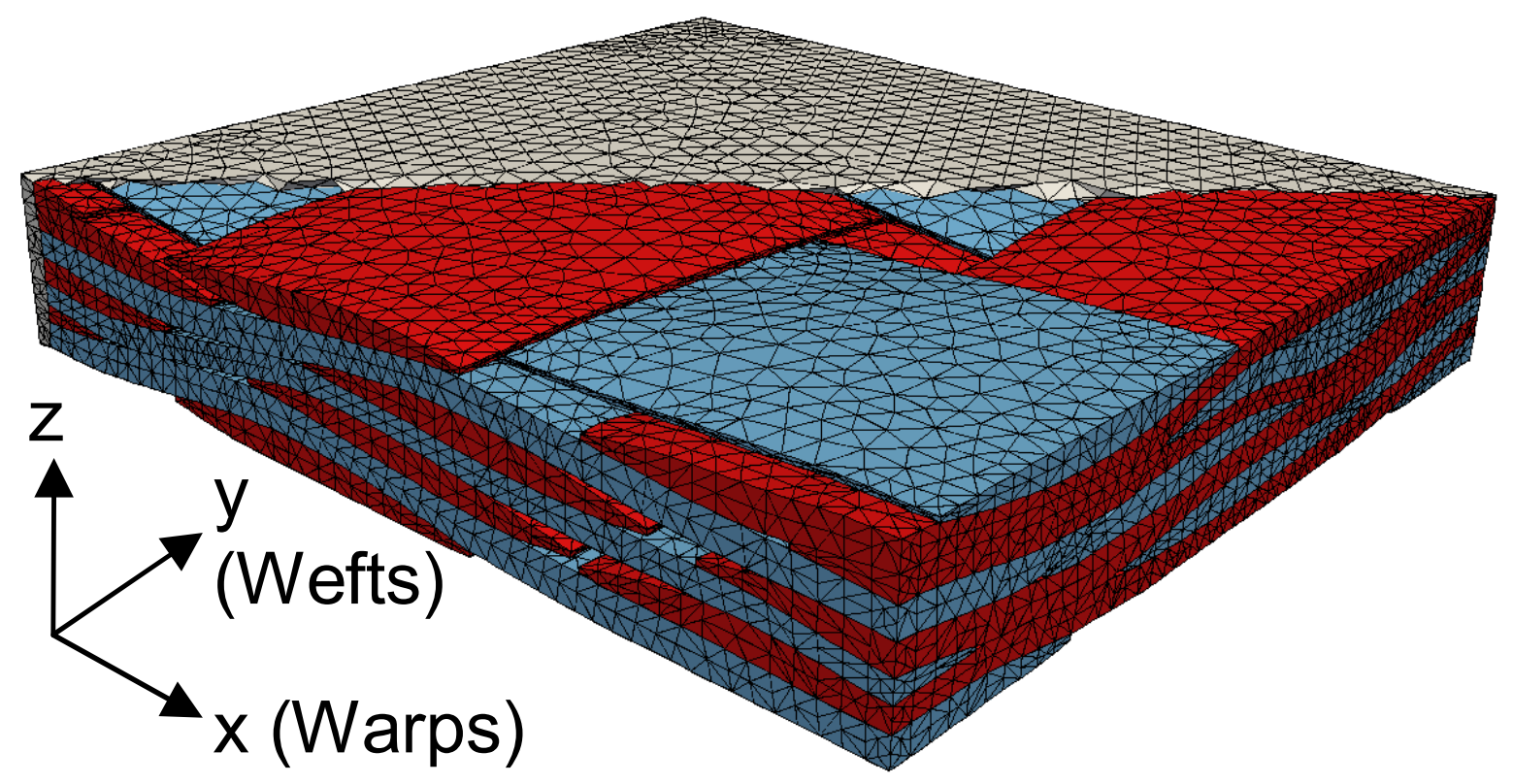

Figure 5: Converged consistent mesh of the RUC with 259000 nodes.

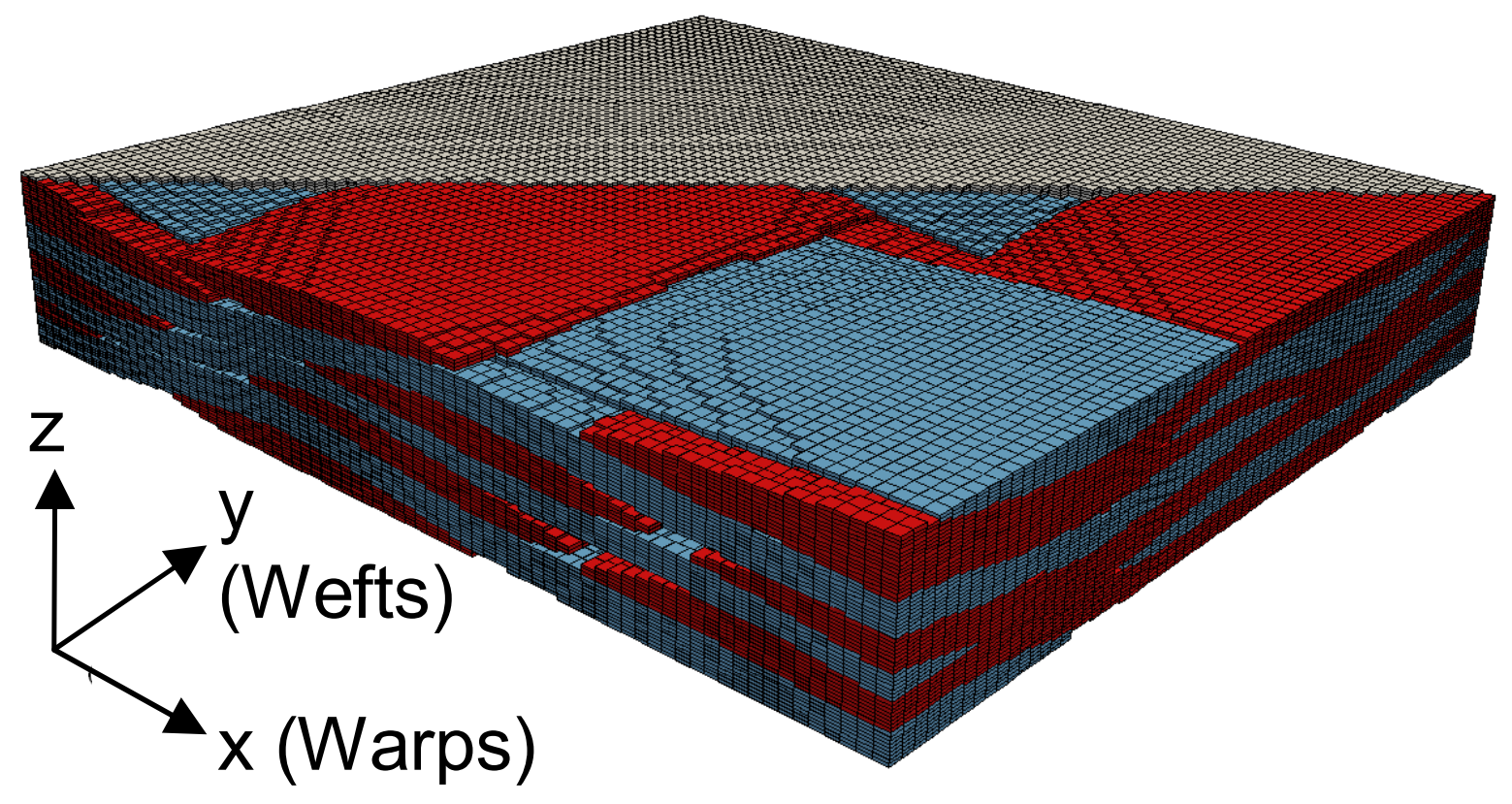

Figure 6: Converged voxel mesh of the RUC with 504000 nodes. 


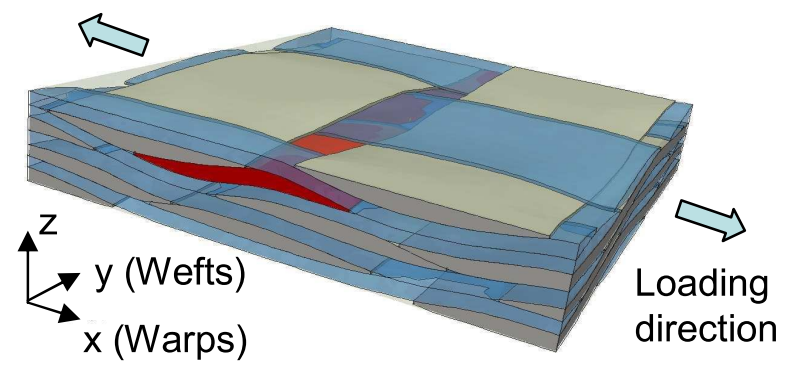

Figure 7: Schematic view of the FE simulation setup. A tensile stress is imposed in the direction of the warps. The weft yarn marked in red is used to study the local stress fields and damage onset.

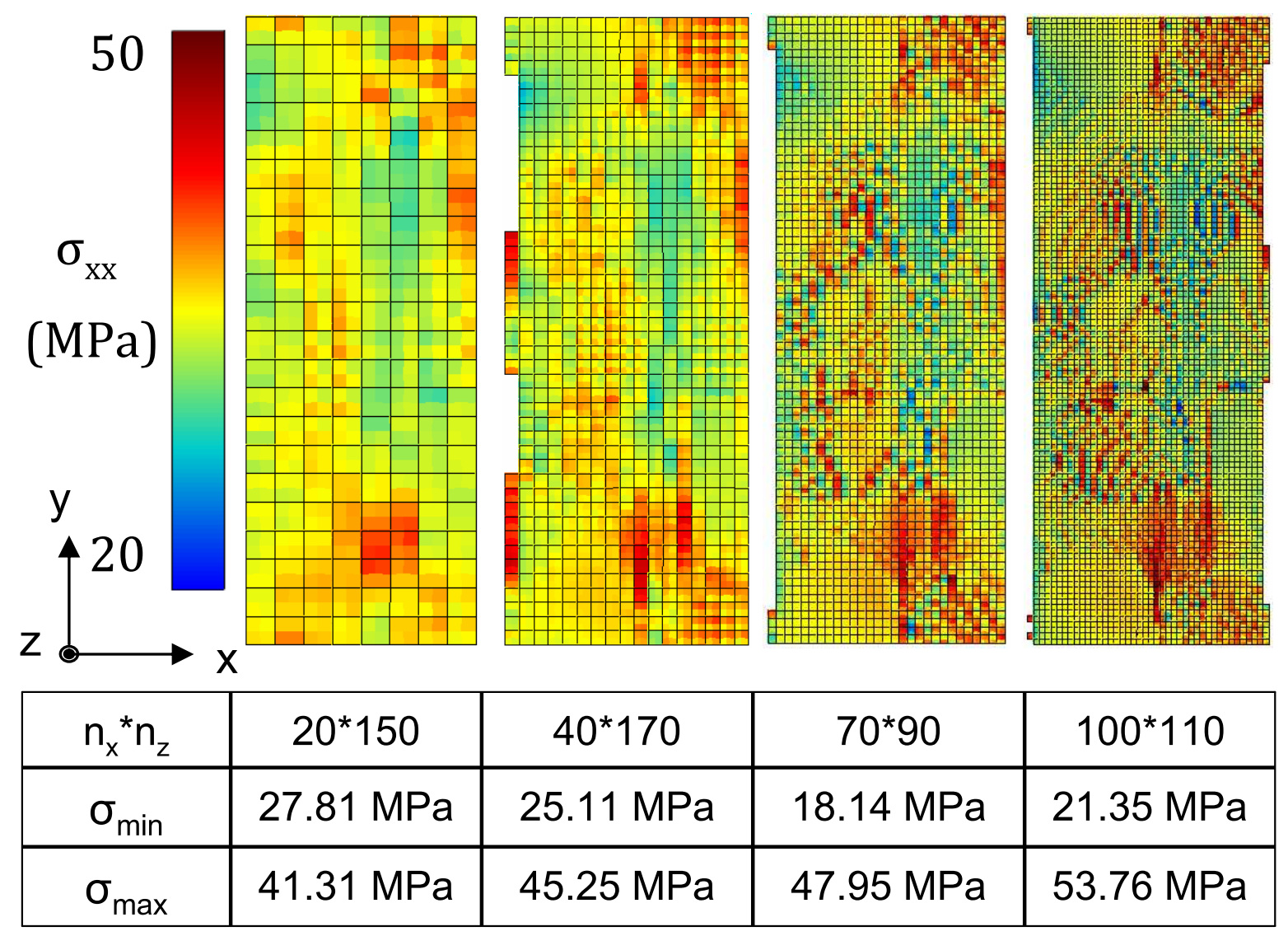

Figure 8: In-plane transverse stress fields on the surface of the weft yarn marked in red in Figure 7 obtained with voxel meshes with different voxel sizes. The maximum and minimum local stress in this yarn are given in the table. 


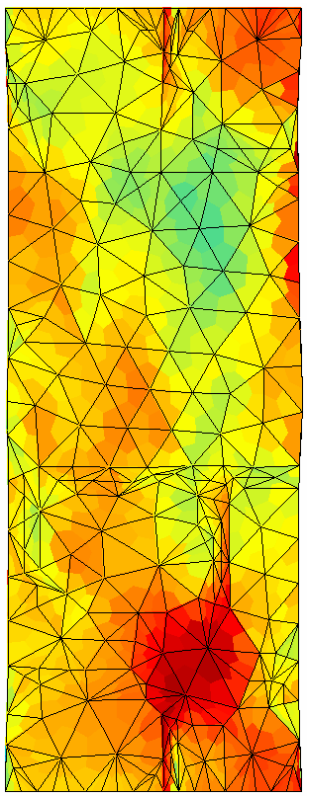

$0.6 \mathrm{~mm}$

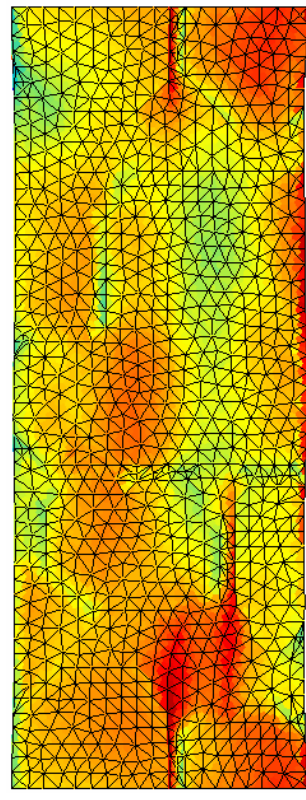

$0.25 \mathrm{~mm}$

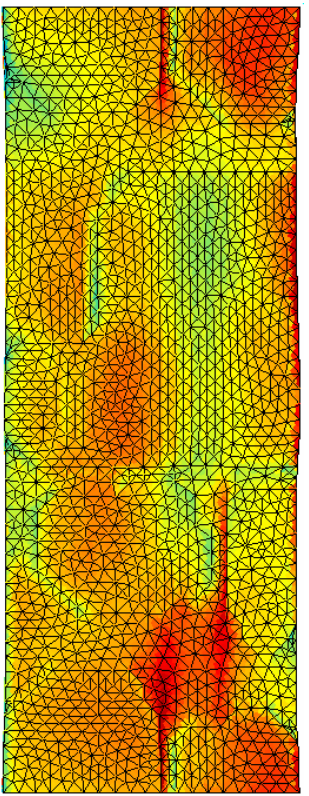

$0.18 \mathrm{~mm}$

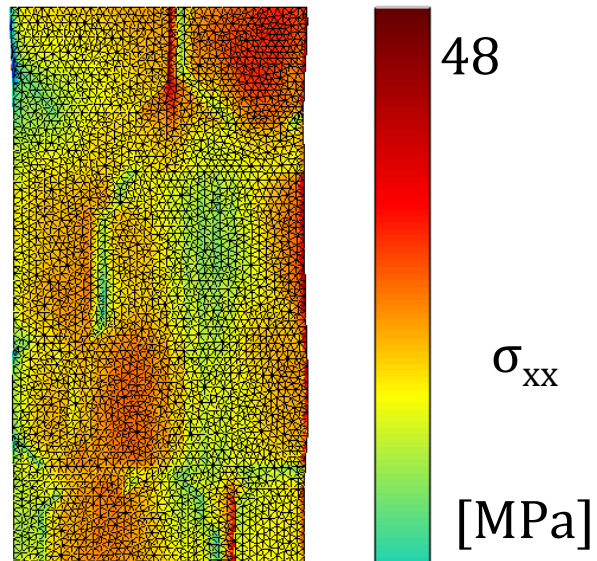

$y$

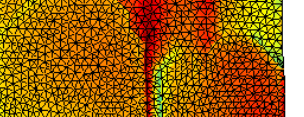

$0.1 \mathrm{~mm}$

Figure 9: In-plane transverse stress fields on the surface of the weft yarn marked in red in Figure 7 obtained with different consistent meshes. The mesh size is indicated under the corresponding stress field.

(a) Voxel mesh

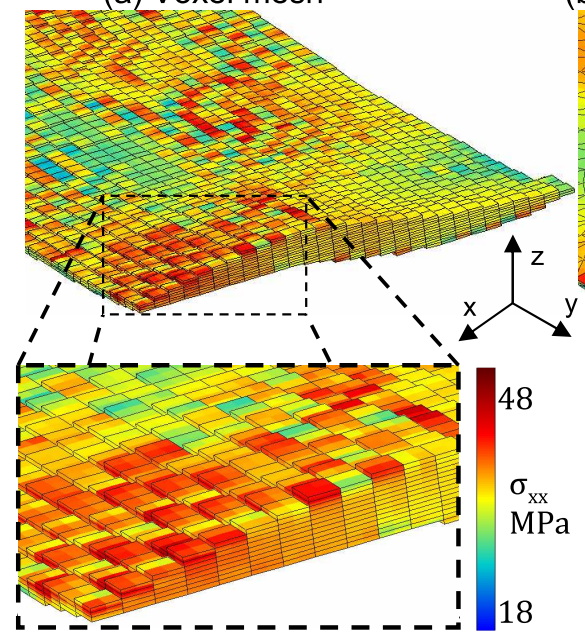

(b) Consistent mesh

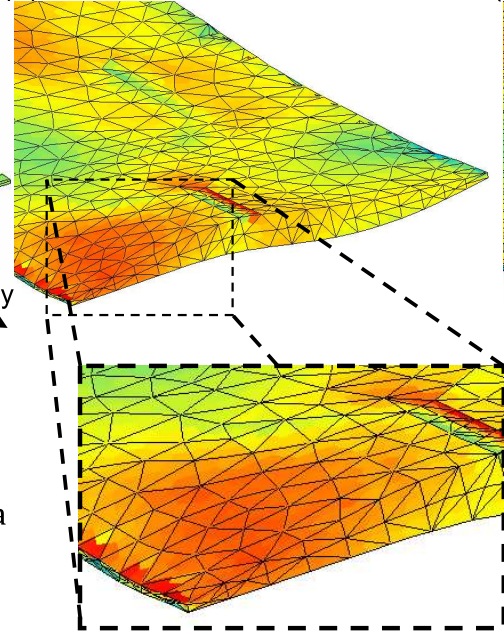

(c) Refined consistent mesh

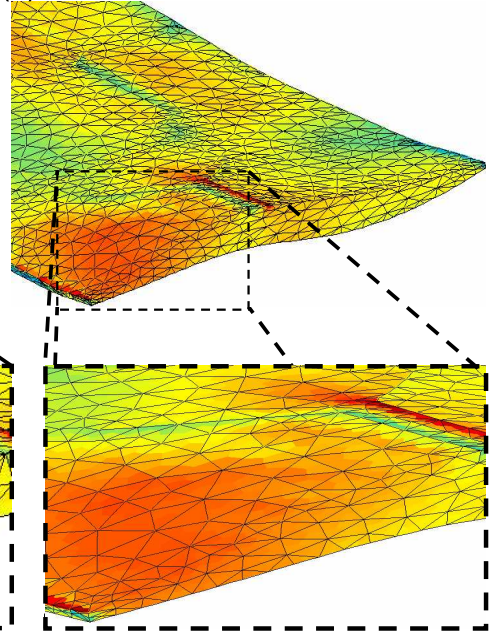

Figure 10: $3 \mathrm{D}$ view of in-plane transverse stress fields in the weft yarn marked in red in Figure 7 obtained with (a) the voxel mesh with $n_{x}=70$, (b) the converged consistent mesh, and (c) the refined consistent mesh. 


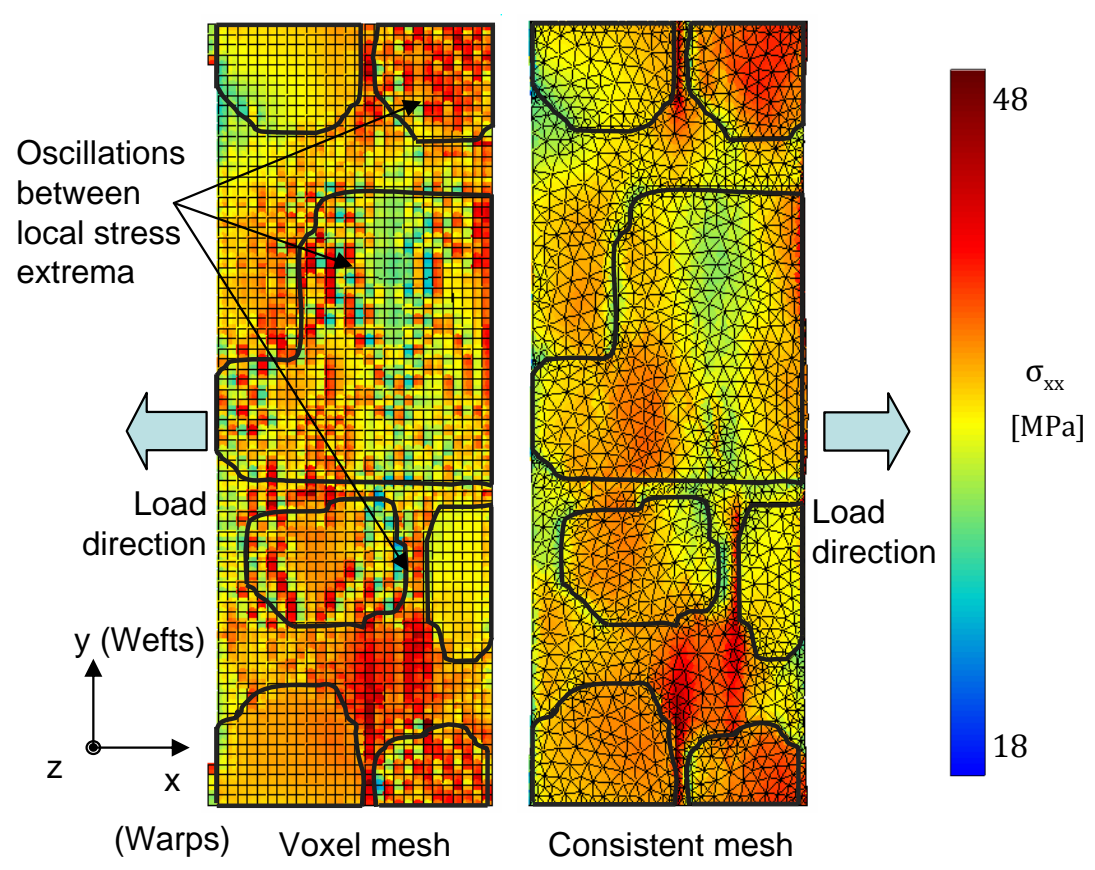

Figure 11: Comparison between the in-plane transverse stress fields on the surface of the weft yarn marked in red in Figure 7 obtained with the voxel mesh with $n_{x}=70$ (left) and the refined consistent mesh (right). The contours of the yarn contact zones are traced in black.

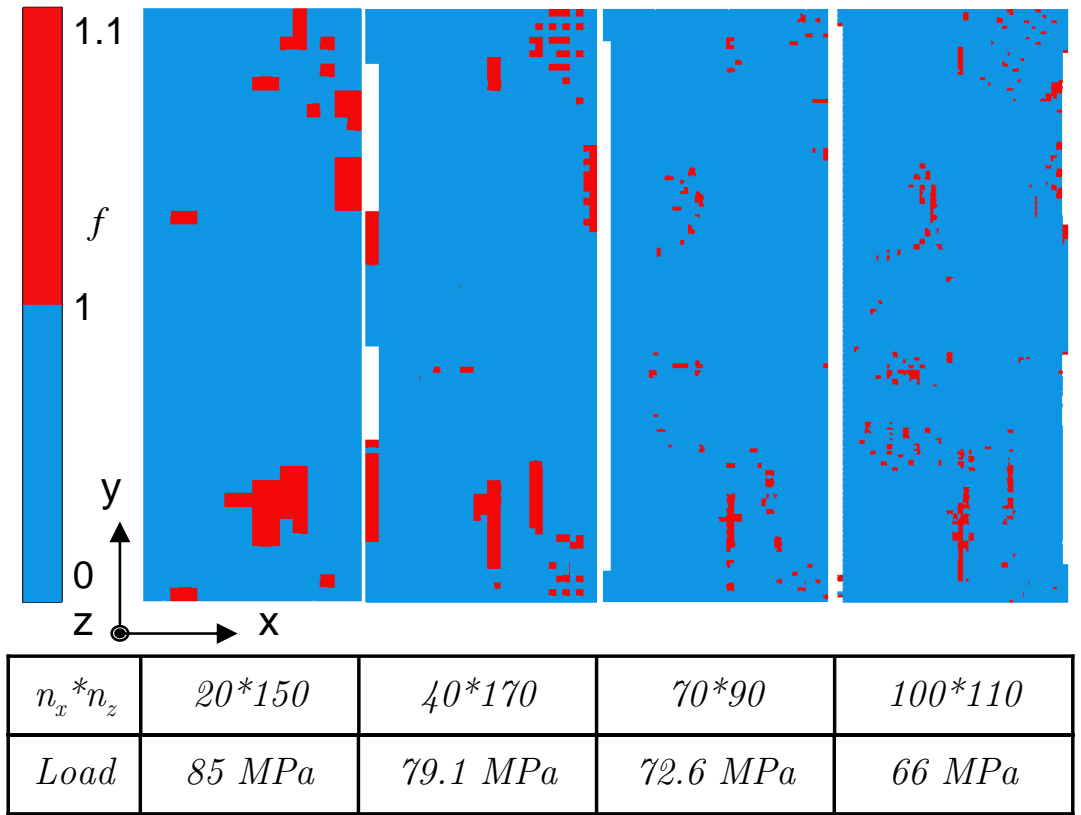

Figure 12: Comparison of the damage locations predicted using the voxel meshes presented in Figure 8. Damage onset prediction is influenced by stress concentrations, regardless of the voxel size. The load required to reach the failure criterion decreases as the voxel density increases. 


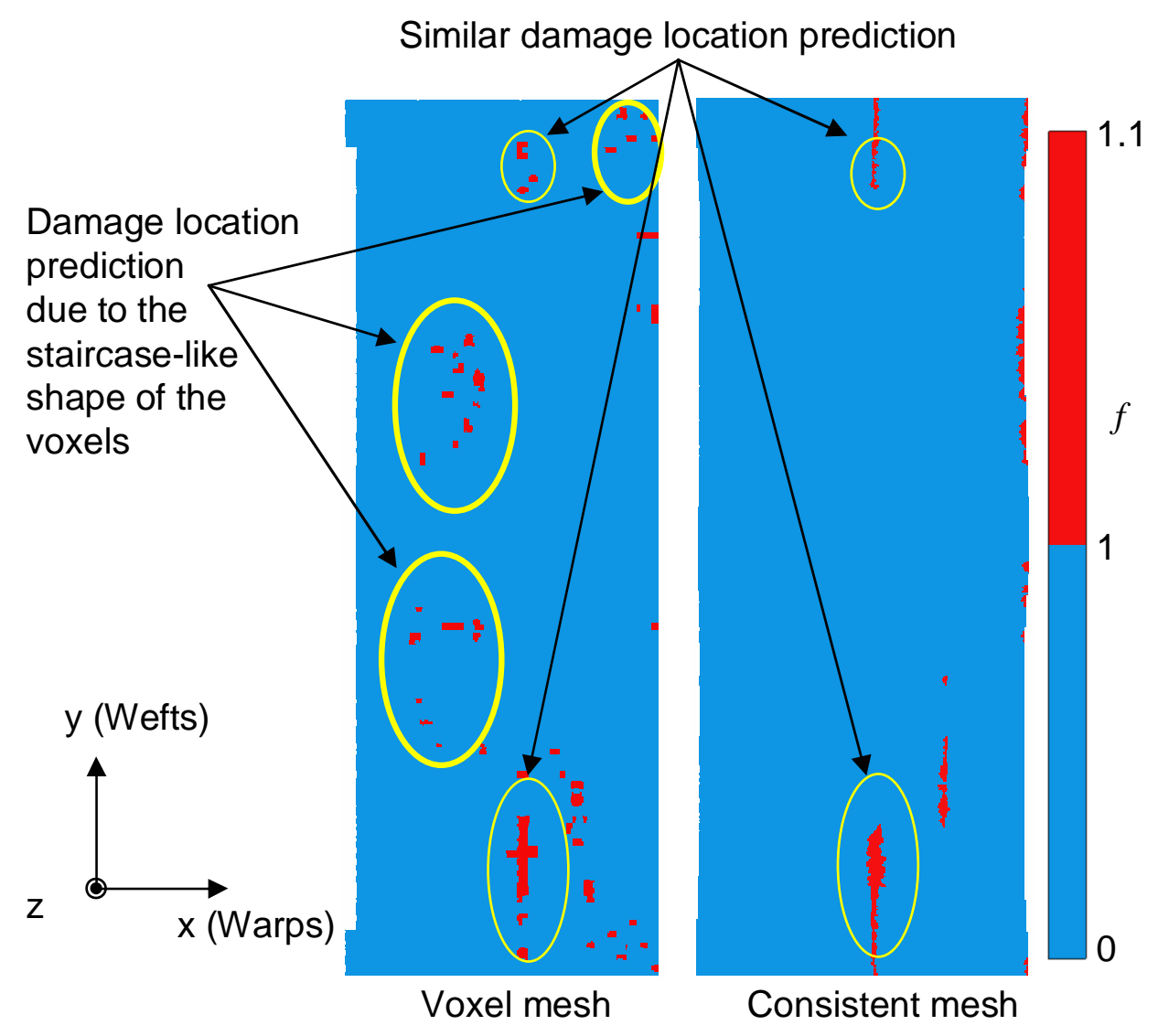

Figure 13: Comparison of the damage locations predicted by voxel and consistent meshes. Similarities and differences are highlighted.

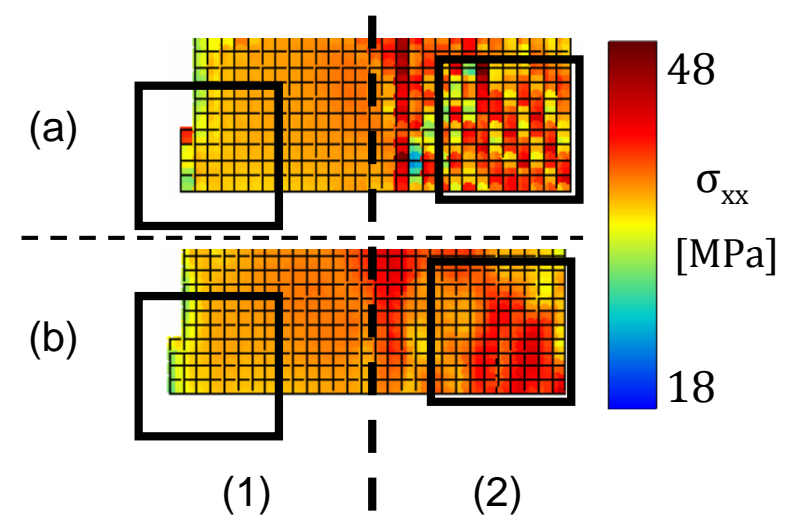

Figure 14: (a) Non smoothed and (b) smoothed voxel stress fields. If the stress concentration is located in only one element (1a), its effect is significantly reduced by smoothing (1b). If the stress concentration extend over several elements in the same zone (2a), smoothing is not sufficient to eliminate their effect $(2 \mathrm{~b})$. 


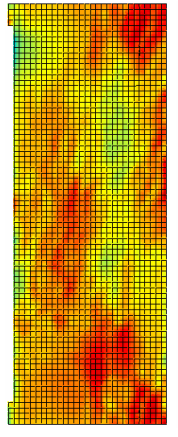

(a)

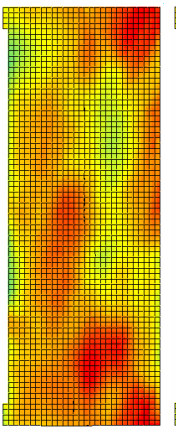

(b)

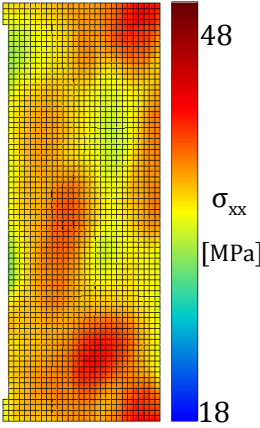

(c)

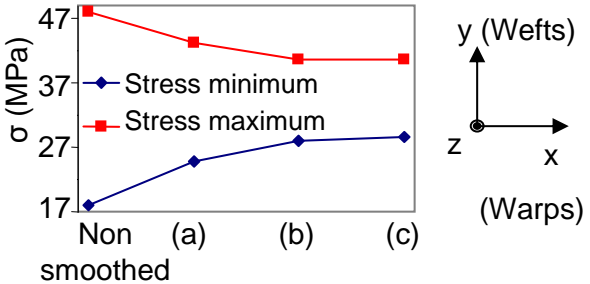

Figure 15: Above: Stress fields obtained with the voxel mesh with $n_{x}=70$ after smoothing over (a) 216, (b) 1000, and (c) 2744 integration points. Below: Evolution of the local stress maximum and minimum in the yarn with the level of smoothing.

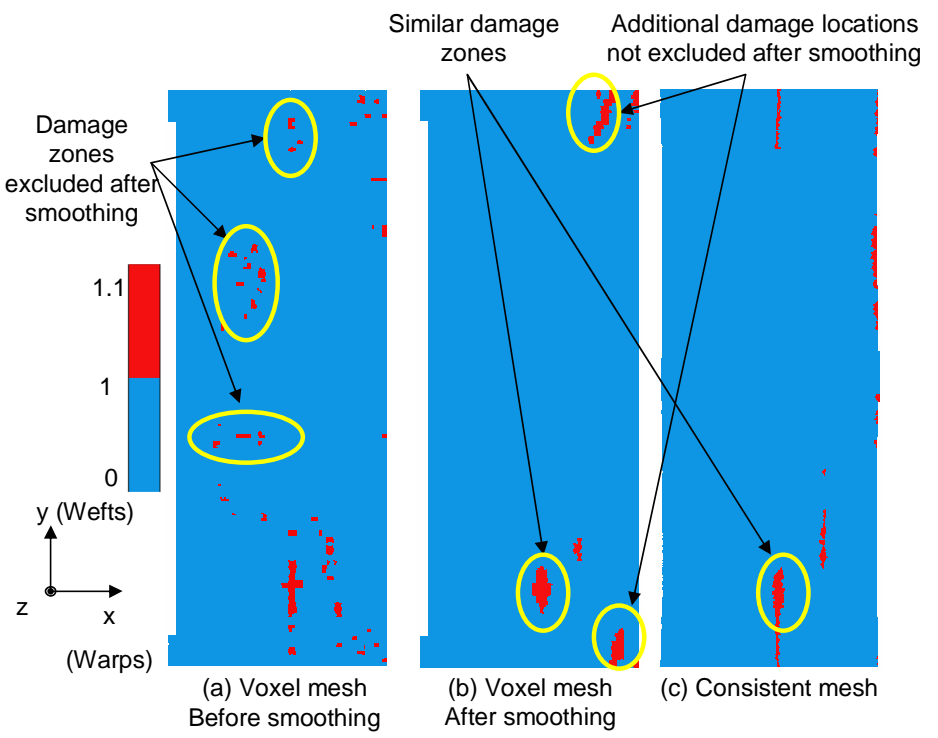

Figure 16: Damage locations predicted with the voxel mesh with $n_{x}=70$ before (a) and after (b) smoothing over 216 integration points in comparison with (c) the predictions obtained from the refined consistent mesh. 